\title{
Environment-Dependent Electron Beam Reduction of Rutile Nanocrystals at High Temperatures
}

\author{
Aram Yoon ${ }^{1,2}$, Jian-Min Zuo ${ }^{1,2 *}$ \\ 1. Materials Science and Engineering, University of Illinois at Urbana Champaign, Urbana, IL, USA. \\ 2. Fredrick Seitz Material Research Laboratory, University of Illinois at Urbana Champaign, Urbana, IL, \\ USA \\ * Corresponding author: jianzuo@illinois.edu
}

Environmental transmission electron microscopy (ETEM) opens a new field for exploring dynamic behavior of the materials in-situ in response to varying experimental stimuli in TEM. The electron beam itself is included as a stimuli; electron interacts with the sample and the environment and thus electron probing of the reacting materials also increase the chance of driving the material far from its steady state reactions. For example, the reduction mechanism evoked by the electron beam in metal oxide [1-3] is often different from the reduction that is solely driven by the chemical potential changes. In order to understand the genuine sample reaction mechanism with the environment, the beam interaction with the sample and the environment has to be addressed first.

Here, we studied the reduction mechanism of rutile $\left(\mathrm{TiO}_{2}\right)$ nanocrystals at high temperatures. To address the electron beam effect, we varied the electron dose rate from low $\left(\sim 10 \mathrm{e}^{-/} \AA^{2} \mathrm{~s}\right)$ to high over $\left.400 \mathrm{e}^{-} / \AA^{2} \mathrm{~s}\right)$ at $550{ }^{\circ} \mathrm{C}$ and recorded the structure change with time. The observation was done using an ETEM (Hitachi 9500, LaB6) operated at $300 \mathrm{kV}$. This instrument combines the sample heating and gas environment with a CMOS-based, high-fidelity and high-transfer rate direct electron detection camera (K2 Summit, Gatan) [4]. The $\mathrm{TiO}_{2}$ nanocrystals of average size $\sim 50 \mathrm{~nm}$ were supported on the $\mathrm{SiN}$ membrane of MEMS heating holder (Blaze, Hitachi Canada) [5,6]. The combination of the stable heating stage at high temperature and the direct electron detection unable us to achieve the atomic resolution at low dose by recording the images with the long exposure time (4-10 s).

The electron beam acts as a strong reducing agent in the TEM vacuum $\left(\sim 10^{-5} \mathrm{~Pa}\right)$. Under the electron beam and in vacuum, the reconstructed the (110) surface structure of $\mathrm{TiO}_{2}$ is shown in Figure 1. Further reduction leads to the formation of the crystallographic shear planes (CSP) inside the nanocrystals (Figure 2 ). With oxygen injection to the column up to the pressure of $3 \times 10^{-3} \mathrm{~Pa}$, the defective CSPs moved or even disappeared, and the nanocrystals surface were also re-oxidized. Once $\mathrm{TiO}_{2}$ nanocrystals were oxidized under the oxygen partial pressure, the surface structure was stable and not affected by the electron beam even at the high dose rate. We also found that the dose rate was more critical than the total dose for the reduction and defect formation of $\mathrm{TiO}_{2}$ nanocrystals. At high dose rate, the electron beam introduced CSPs to the nanocrystals in a few seconds. At low dose rate below the empirical threshold rate of $100 \mathrm{e}^{-}$ $/ \AA^{2} / \mathrm{s}$, the defects did not form regardless total illumination time. Comparing with the control experiments performed at room temperature, we concluded the reduction mechanism is governed by the competition between the reduction rated by the electron beam and the recovery rate at high temperatures [7]. 
References:

[1] DJ Smith, MR McCartney and LA Bursill, Ultramicroscopy 23 (1987), p. 299.

[2] MI Buckett and LD Marks, Surface Science 232 (1990), p. 353.

[3] MR McCartney and DJ Smith, Surface Science 250 (1991), p. 169.

[4] G McMullan et al., Ultramicroscopy 147 (2014), p. 156.

[5] JY Howe et al., Microscopy and Microanalysis 23 (2017), p. 66.

[6] AD Sediako et al., Energy \& Fuels 33 (2019), p. 1582.

[7] Work supported by DOE BES (Grant No. DEFG02-01ER45923).
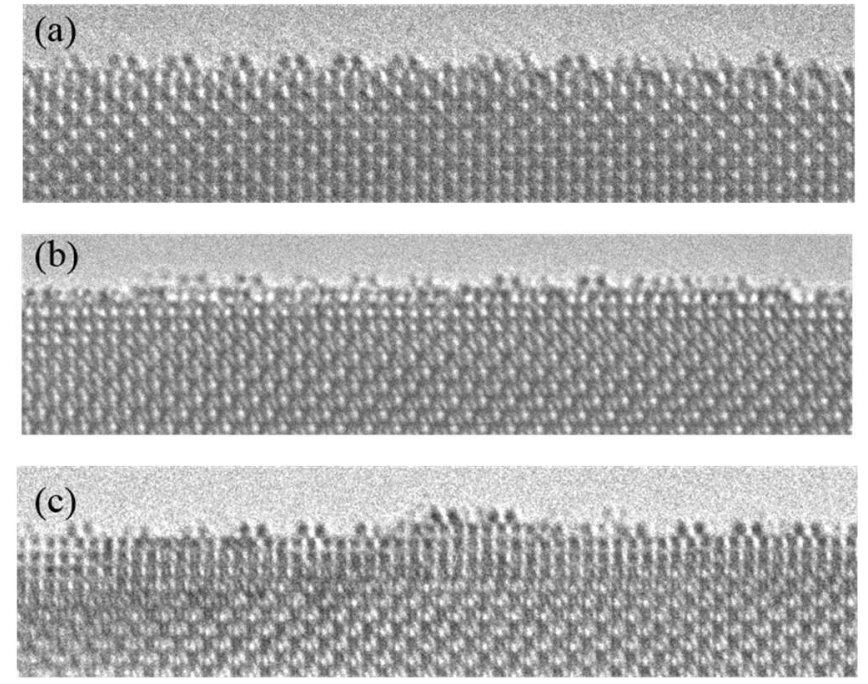

Figure 1. The progression of the $\mathrm{TiO}_{2}(110)$ surface reconstruction in vacuum with the electron dose rate of $580 \mathrm{e}^{-} / \AA^{2} \mathrm{~s}$ (a) at the beginning, (b) after 4 minutes, and (c) after 14 minutes.
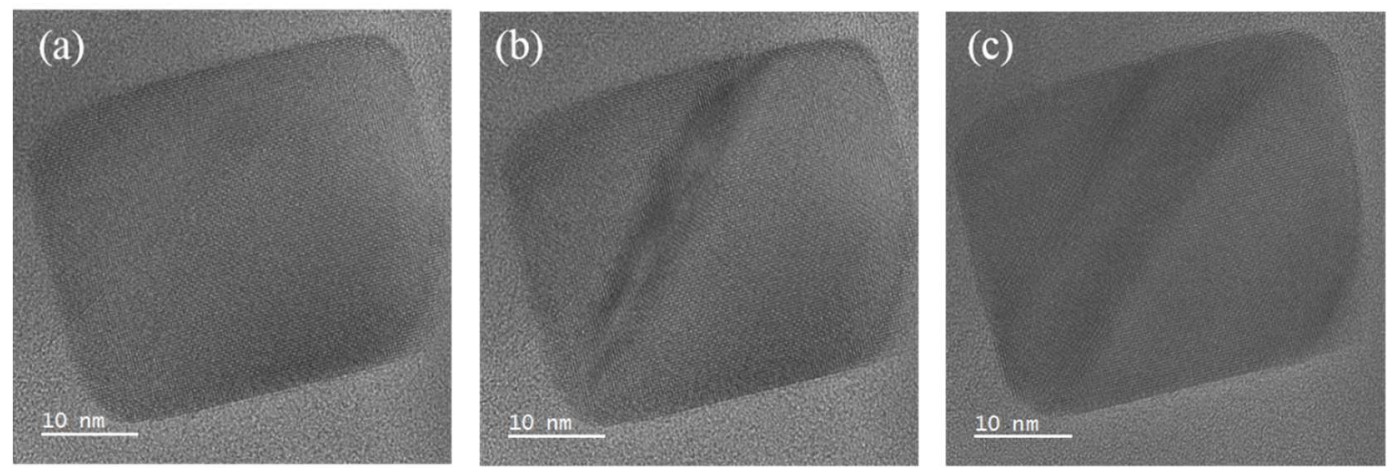

Figure 2. Crystallographic shear plane (CSP) formation of $\mathrm{TiO}_{2}$ nanocrystal in vacuum under e-beam illumination with the following conditions: the dose rate was $420 \mathrm{e}^{-} / \AA^{2} \mathrm{~s}$, and the exposure time was 4 seconds for each image. (a) Pristine $\mathrm{TiO}_{2}$ particle at the beginning (b) after 25 seconds of illumination. (c) The CSP moved after the oxygen treatment. 